\title{
Dificuldades enfrentadas pelo enfermeiro na prevenção da obesidade infantil: uma revisão integrativa
}

\author{
Difficulties faced by nursing to prevent children's obesity: an \\ integrative review
}

Flávia Gonçalves Isabel Barbone`; Vander Lúcio Mendes’; Heuler Souza Andrade’

\begin{abstract}
'Graduados em Enfermagem pela Universidade do Estado de Minas Gerais UEMG/Divinópolis/MG/Brasil.
\end{abstract}

\begin{abstract}
Resumo
Introdução: A obesidade atualmente é considerada um dos principais problemas de saúde pública e vem aumentando progressivamente entre crianças. O enfermeiro possui um papel relevante na abordagem nutricional e no acompanhamento do crescimento e desenvolvimento infantil. Objetivo: Descrever as principais dificuldades do enfermeiro na prevenção da obesidade infantil. Metodologia: Trata-se de uma revisão integrativa da literatura. Foram utilizados os bancos de dados MEDLINE, LILACS e BDENF. Foram incluídos artigos indexados e disponíveis, publicados entre os anos de 2010 e 2019, nos idiomas inglês e português. Foram excluídos artigos não disponíveis em texto completo, que não correspondiam a temática em questão, resenhas, teses, dissertações e monografias. Resultados: De 70 artigos, apenas 07 artigos corresponderam ao objetivo proposto. Identificou-se que a maior dificuldade para abordagem nutricional da criança está relacionada aos pais $(71,4 \%)$ que não consideram o excesso de peso como um agravante em saúde. E em relação ao acompanhamento do crescimento e desenvolvimento infantil, a maior dificuldade do enfermeiro (42,8\%) está relacionada aos problemas de infraestrutura para prestação de serviços e na falta de equipamentos para prestação dos cuidados. Conclusão: É necessário criar estratégias de saúde que envolvam os pais e enfermeiro de forma dinâmica e educativa para um melhor entendimento dos fatores de riscos relacionado à obesidade infantil. Por parte da gestão dos serviços de saúde, é fundamental, oferecer condições e equipamentos para os profissionais de enfermagem e equipe de saúde para exercerem atenção integral à criança com qualidade e eficiência.
\end{abstract}

Palavras-chave: Obesidade Infantil; Enfermagem; Prevenção. 


\begin{abstract}
Introduction: Obesity is currently considered one of the main public health problems and has been increasing progressively among children. The nurse has a relevant role in the nutritional approach and in monitoring child growth and development. Objective: To describe the nurse's main difficulties in preventing childhood obesity. Methodology: This is an integrative literature review. The MEDLINE, LILACS and BDENF databases were used. Indexed and available articles, published between 2010 and 2019, in English and Portuguese were included. Articles not available in full text, which did not correspond to the subject in question, reviews, theses, dissertations, and monographs were excluded. Results: Out of 70 articles, only 07 articles met the proposed objective. It was identified that the greatest difficulty in the child's nutritional approach is related to parents (71.4\%) who do not consider being overweight as a health aggravating factor. And in relation to monitoring child growth and development, the greatest difficulty for nurses (42.8\%) is related to infrastructure problems for the provision of services and the lack of equipment to provide care. Conclusion: It is necessary to create health strategies that involve parents and nurses in a dynamic and educational way for a better understanding of the risk factors related to Childhood Obesity. On the part of the management of health services, it is essential to offer conditions and equipment for nursing professionals to provide comprehensive care to children with quality and efficiency.
\end{abstract}

Keywords: Childhood Obesity; Nursing; Prevention.

Recebido em: 07-07-2020 Publicado em:29-10-2020

\title{
Autor correspondente
}

Flávia Gonçalves Isabel Barbone

Endereço: Rua Mar de Espanha, n 60-Belvedere II, Divinópolis-MG.

E-mail:flavia1379@yahoo.com.

\section{Introdução}

A obesidade é definida como um distúrbio nutricional epidemiológico com prevalência crescente, caracterizado pelo alto índice de gordura corporal ? Atualmente, é considerado um dos principais problemas de saúde pública, e que eleva os riscos para o adoecimento na vida adulta ${ }^{2-3}$. Segundo a Organização Mundial da Saúde (OMS), estima-se que em 2025 há em torno de 70 milhões de crianças obesas no mundo 4 .
As causas e fatores de riscos são vários, incluindo genéticos, emocionais, psicossociais, socioeconômicos, ambientais e culturais. Mas, os hábitos de vida que levam a alimentação mal balanceada e a falta de atividade física são os maiores agravantes da obesidade infantil ${ }^{5}$. Com isto, o excesso de peso na infância ao longo do tempo aumenta as chances da ocorrência de múltiplas doenças, tais como: cardiovasculares, cancerígenas, diabetes mellitus (DM), hipertensão arterial sistêmica (HAS), 
acidente vascular encefálico (AVE), entre outras comorbidades 1, 3,5.

O enfermeiro como um educador em saúde possui um papel relevante na participação nutricional e no acompanhamento do crescimento e desenvolvimento infantil ${ }^{6}$. Este, em conjunto com a equipe multidisciplinar em saúde possui a responsabilidade em realizar ações em educação em saúde como ferramenta para auxiliar na prevenção, manutenção e restauração da saúde ${ }^{7}$.

A transição nutricional devido às mudanças do estilo de vida e o aumento da obesidade na infância, tanto no sexo masculino tanto no sexo feminino, tornase um grande desafio para a saúde pública. Neste contexto, o acompanhamento e a avaliação do enfermeiro nas consultas de puericultura é de suma importância em cada fase da infância. A orientação sobre alimentação saudável e acompanhamento do crescimento e desenvolvimento psicomotor, são uma das principais ações de rotina nas consultas de puericultura ${ }^{8-}$ 10 .

Por essas razões, torna-se necessário identificar as principais dificuldades enfrentadas pelo enfermeiro para realizar um aconselhamento nutricional e acompanhar o crescimento e desenvolvimento da criança. Para assim, aumentar as possibilidades de intervenção da obesidade infantil com uma assistência mais efetiva e com qualidade no contexto da atenção básica do Sistema único de Saúde "?.

Neste quesito, surge a seguinte questão norteadora do estudo: "Quais as principais dificuldades do enfermeiro na prevenção da obesidade infantil? ". Ao detectar as principais fontes dessa problemática, será possível o enfermeiro analisar qual a melhor forma de agir preventivamente a este público desde as consultas do pré-natal a puericultura, bem como acompanhar o estado nutricional da criança em cada fase da infância, melhorar o conhecimento dos familiares sobre alimentação saudável e a importância da prática de atividades físicas. Assim, o objetivo desse estudo foi descrever as principais dificuldades do enfermeiro na prevenção da obesidade infantil.

\section{Metodologia}

Trata-se uma revisão integrativa da literatura, um método sintetizado que permite a análise de um determinado tema e possibilita a síntese de múltiplos estudos publicados. A revisão integrativa consiste em seis momentos: elaboração da temática, busca de pesquisas na literatura, coleta de dados, análise crítica e integrativa dos estudos selecionados, discussão dos resultados, e por último, a apresentação dos resultados da temática 12.

A busca pela literatura ocorreu na Biblioteca Virtual em Saúde (BVS), utilizou-se o banco de dados da MEDLINE (Medical Literature Analysis and Retrieval System Online), BDENF (Banco de Dados em Enfermagem), LILACS (Literatura Latino-americana e do Caribe em Ciências da Saúde). Utilizou-se os seguintes descritores: "obesidade infantil" AND "prevenção" AND "enfermagem".

As buscas de dados foram limitadas pela data de publicação, só foram incluídos artigos publicados entre 2010 e 2019. No total, foram encontradas 70 publicações disponíveis, nos idiomas inglês e português. A coleta de dados foi realizada nos meses de maio, junho e julho do ano 
de 2019. Como assunto principal foram filtrados: obesidade; pediátrica; promoção da saúde; papel do profissional de enfermagem.

Foram utilizados os seguintes critérios de inclusão: artigos indexados e disponíveis; publicações referentes as dificuldades da enfermagem na prevenção e tratamento da obesidade infantil referente a faixa etária de $\mathrm{O}$ a 12 anos, conforme descrito Estatuto da Criança e do Adolescente (ECA) - Lei 8069/90 13. Os critérios de exclusão da amostra: literatura cinzenta que não correspondem a temática em questão; resenhas; citações; teses, dissertações e monografias.

A seleção das publicações foi realizada por meio da leitura de títulos e resumos para exclusão de duplicidade e daqueles que não interessam. Em seguida, leitura na íntegra referente as publicações que correspondiam a temática e objetivo proposto para extração de dados. A construção das categorias foi definida pelos autores para melhor divisão e organização dos fatores dificultadores encontrados nas publicações selecionadas.
Em virtude da natureza da pesquisa, por meio de dados de domínio público, não foi necessário submeter ao Comitê de Ética em Pesquisa. Mas, foram respeitados todos os preceitos éticos estabelecidos pela Resolução n 466/2012, conforme descrito no Conselho Nacional de Saúde ${ }^{14}$.

\section{Resultados}

$\mathrm{Na}$ presente revisão integrativa, o processo de seleção das publicações foi apresentado detalhadamente no organograma 1, foram encontrados na Biblioteca Virtual em Saúde no total de 70 publicações. No primeiro momento, utilizou-se os métodos de inclusão e exclusão, foram filtrados em: 32 publicações. No segundo momento, foi realizado leitura na íntegra para extração de dados, e aqueles que contemplaram a pergunta norteadora e corresponderam o objetivo proposto, foram no total de sete publicações disponíveis. 
ORGANOGRAMA 1- Identificação, seleção e inclusão das publicações na Revisão

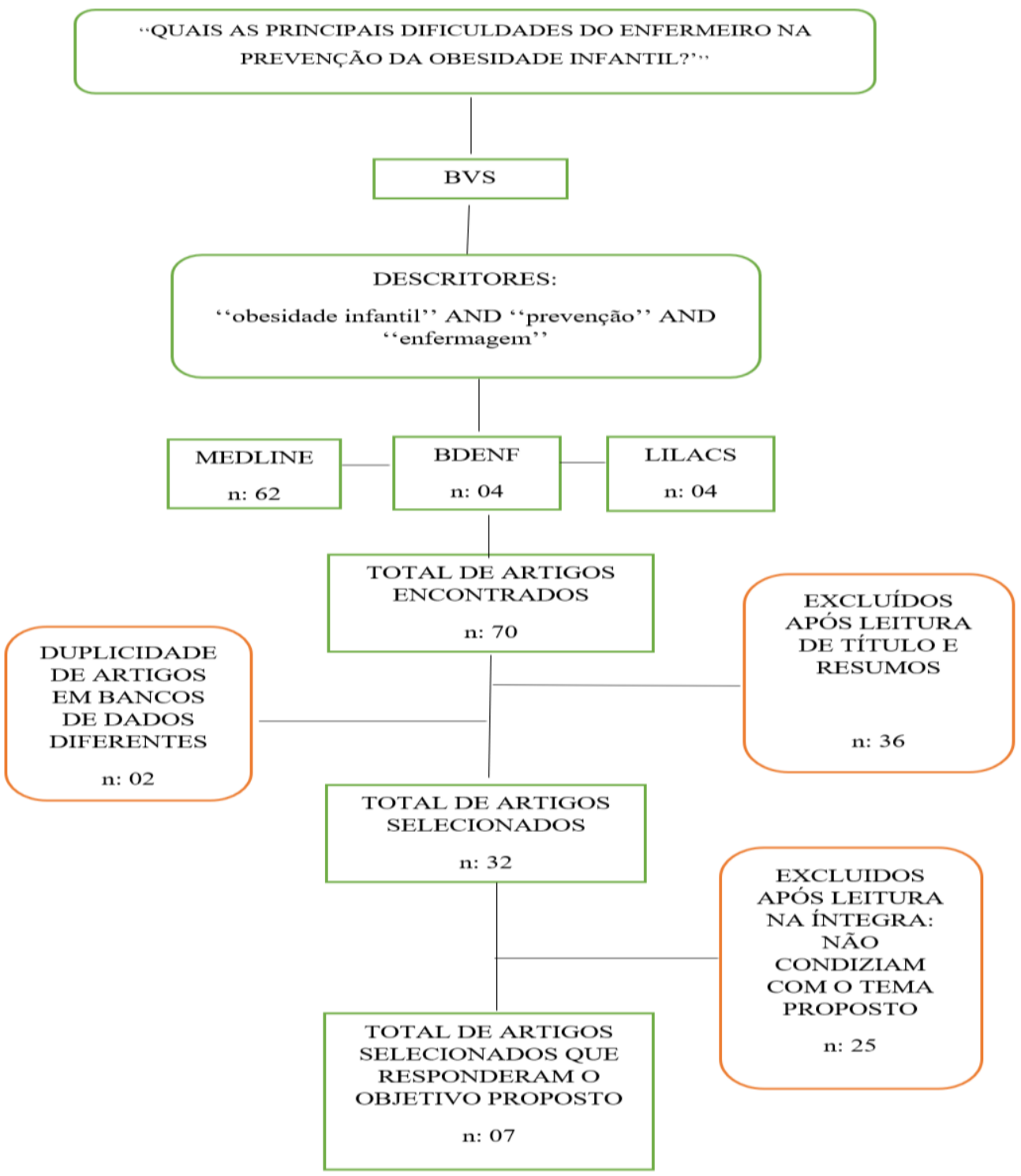

Fonte: Elaborado pelos autores, 2019.

Para construção do estudo, análise e interpretação dos sete artigos selecionados, foi elaborado um quadro (Quadro 1), com as seguintes categorias: título; autor e ano de publicação; periódico e objetivo. $O$ ano de 2013 foi o de maior número de publicações observadas, com três (42,8\%) dos artigos. 


TíTULO AUTORES PERIÓDICO OBJETIVO

Barreiras para abordar problemas de peso na infância e como superálos

\section{WARREN, N. et al.}

(2017)

A experiência do prestador de cuidados de saúde com pais de crianças com sobrepeso

e obesidade: uma análise qualitativa
ANTI, Eliza et al.

iza et al.

\section{(2016)}

\author{
Nursing \\ Children and
Young People
}

Identificar as barreiras que

s enfermeiros enfrentam

para abordar questões de peso infantil com a família.
Facilidades

dificuldades

enfermeiros no cuidar

da alimentação infantil

na atenção básica

e dos al.

(2015)

MOURA, Mayara Águida Porfírio, et

Obesidade Infantil: os desafios para os enfermeiros
PENN, Sarah et al. (2014)

\section{Journal Pediatric Health Care}

Explorar a experiência dos prestadores de cuidados de saúde no ambiente ambulatorial ao trabalharem com pais de crianças com sobrepeso e obesidade.
O Mundo da Compreender as Saúde, São facilidades e dificuldades Paulo.

no enfermagem, relacionado à alimentação infantil no cotidiano do enfermeiro na rede de atenção básica

Nursing
Children \&
Young People

Identificar os principais desafios para os enfermeiros na obesidade infantil. 
Registros de CORGOZINHO,

enfermagem e o Juliana Nunes

enfoque na prevenção Costa, et al.

da obesidade infantil

(2013)

Obstáculos

na

prevenção do sobrepeso

e obesidade nos

cuidados de saúde a

crianças na Suécia

ISMA, Gabriela E. et al.

(2013)
RECOM- Revista de Enfermagem do Centro Oeste Mineiro
Analisar as anotações voltadas para a prevenção da obesidade infantil, conforme preconizado pelo

Ministério da Saúde.

\author{
BMC- Family Elucidar os obstáculos \\ Practice enfrentados pelo \\ enfermeiro de seu \\ trabalho preventivo com \\ sobrepeso e obesidade \\ infantil na atenção à \\ saúde da criança.
}

Influência das percepções no estudo de enfermagem para prevenir a obesidade infantil
QUELLY, Susan B. The Journal of et al

(2013)
Determinar a influência de percepções da enfermeira-escola (autoeficácia, benefícios percebidos e barreiras percebidas) sobre a participação em práticas de no nível individual da criança e da escola.

Fonte: Elaborado pelos autores, 2019.

O primeiro estudo dessa categoria $^{15}$, aponta que os pais são os principais dificultadores, pois, para muitos o excesso de peso da criança não é um fator relevante para o adoecimento. Além disso, muitos pais desinteressam pelas atividades educativas relacionadas a alimentação saudável na infância e demonstram certa resistência na mudança dos hábitos alimentares. Outro quesito importante destacado no estudo, é falta de recursos nas Unidades de Atenção Primária e a falta de preparo dos profissionais de saúde ao abordarem os familiares sobre o assunto obesidade infantil.

O segundo estudo dessa categoria $^{16}$, destaca que o consumo diário de alimentos industrializados, o estilo de vida sedentário, a resistência dos pais perceberem os maus hábitos alimentares 
e a ausência destes no cotidiano da criança, são os principais complicadores para o sucesso das ações educativas do enfermeiro e de outros profissionais da saúde na abordagem preventiva.

O terceiro estudo dessa categoria ${ }^{17}$, descreve as facilidades e dificuldades do enfermeiro da Atenção Básica na prestação dos cuidados em relação à alimentação infantil em seu cotidiano. Este estudo ressalta que os aspectos sociais (inexperiência maternal; mãe menor de idade; baixa escolaridade da mãe; pais ausentes nas atividades educativas sobre alimentação saudável), culturais (desmame precoce; resistência da percepção materna ou dos familiares visualizarem o excesso de peso como um problema de saúde) e econômicos (mães usam o benefício da Bolsa Família para outras finalidades ao invés de investirem na nutrição da criança) são as principais dificuldades no aconselhamento nutricional infantil.

○ quarto estudo dessa categoria ${ }^{18}$, ressalta que a maior dificuldade que o enfermeiro enfrenta em relação aos cuidados e tratamento da obesidade infantil está no recrutamento dos pais e familiares nas intervenções para obesidade. E também, a negação dos pais e responsáveis perceberem que o filho está sobrepeso/obeso. Logo, muitos destes encontram-se acima do peso, consomem alimentos industrializados diariamente, possuem um estilo de vida sedentário e ignoram o aconselhamento dos profissionais de saúde para um estilo de vida saudável.

- quinto estudo dessa categoria19, descreve que nas consultas de enfermagem referente a faixa etária de zero a dois anos, as anotações de enfermagem não correspondem corretamente a preconização do Ministério da Saúde. Ou seja, as evoluções do enfermeiro nas consultas de puericultura não estão claramente descritas, faltando informações relevantes sobre o aspecto nutricional da criança. Dessa forma, além de dificultar a continuidade do trabalho interdisciplinar, há uma negligência nas informações referentes a prevenção de obesidade infantil.

O sexto estudo dessa categoria ${ }^{20}$, referese a falta de capacitação dos enfermeiros na abordagem sobre o excesso de peso dos filhos com os pais. Além disso, a falta de recursos, diretrizes e uma gestão em saúde deficiente na Atenção Primária, tornam-se outros obstáculos para os enfermeiros agirem preventivamente contra a obesidade infantil.

O sétimo estudo dessa categoria ${ }^{21}$, descreve sobre o enfermeiro que atua no ambiente escolar e ressalta que a falta de recursos na escola para triagem do Índice de Massa Corporal (IMC); apoio ineficiente dos pais, dos administradores da gestão da Atenção Primária e dos professores no acompanhamento do crescimento e desenvolvimento infantil e a falta de dados na evolução de enfermagem, causam um impacto negativo na qualidade da assistência de enfermagem.

São múltiplos fatores que dificultam o trabalho do profissional enfermeiro nas ações preventivas da obesidade infantil. As dificuldades encontradas em cada estudo, foram organizadas e divididas em duas categorias: "Dificuldades para abordagem nutricional da criança" e "Dificuldades para acompanhamento do crescimento e desenvolvimento infantil". $\mathrm{Na}$ primeira, a dificuldade "Resistência dos pais em relação ao excesso de peso da criança como um problema de saúde" foi a de maior ocorrência, aparecendo em cinco $(71,4 \%)$ artigos entre os pesquisados. $\mathrm{Na}$ segunda, os "Problemas de infraestrutura para prestação de serviços" 
e a "Falta de equipamentos e impressos para prestação dos cuidados e tratamento" apareceram em três (42,8\%) dos artigos observados. Demais dificuldades encontradas nos artigos foram subdividas (Tabela 1), conforme a categoria a seguir:

Tabela 1. Dificuldades enfrentadas pelo enfermeiro na prevenção da obesidade infantil citadas em cada categoria.

\begin{tabular}{|c|c|c|}
\hline Dificuldades & $\mathrm{N}$ & $\%$ \\
\hline \multicolumn{3}{|l|}{ Realizar a abordagem nutricional da criança } \\
\hline $\begin{array}{l}\text { Resistência dos pais em relação ao excesso de peso da criança } \\
\text { como um problema de saúde }\end{array}$ & 5 & 71,4 \\
\hline Pais ausentes no cotidiano do filho & 4 & 57,1 \\
\hline Consumo de alimentos industrializados & 3 & 42,8 \\
\hline Estilo de vida sedentário & 3 & 42,8 \\
\hline Crenças & 2 & 28,6 \\
\hline $\begin{array}{l}\text { Inexperiência maternal, mãe menor de idade, e baixa } \\
\text { escolaridade }\end{array}$ & 1 & 14,3 \\
\hline Desmame precoce & 1 & 14,3 \\
\hline Bolsa Família para outras finalidades & 1 & 14,3 \\
\hline \multicolumn{3}{|l|}{ Acompanhamento do crescimento e desenvolvimento infantil } \\
\hline Problemas de infraestrutura para prestação de serviços & 3 & 42,8 \\
\hline $\begin{array}{l}\text { Falta de equipamentos e impressos para prestação dos } \\
\text { cuidados e tratamento }\end{array}$ & 3 & 42,8 \\
\hline Evolução de Enfermagem com falta de dados relevantes & 2 & 28,6 \\
\hline Falta de capacitação dos profissionais de saúde & 2 & 28,6 \\
\hline Gestão em saúde e diretrizes ineficientes & 2 & 28,6 \\
\hline
\end{tabular}

Fonte: Fonte: Elaborado pelos autores, 2019.

\section{Discussão}

Ao analisar os resultados notou-se que a principal dificuldade do enfermeiro para realizar a abordagem nutricional na infância está em lidar com os pais/ responsáveis em relação ao excesso de peso do filho. A maioria dos estudos analisados citou que os pais subestimam a obesidade, não a considerando como um agravante em saúde ao delongo dos anos. A maioria dos responsáveis da criança procuram os serviços de saúde devido a outras finalidades. Raramente, são devido ao excesso de peso na infância. 
Na visão dos pais, a obesidade infantil não é considerada um problema de saúde relevante, e sim, um sinal de saúde e bem cuidada ${ }^{15,18,20 .}$

As ações educativas sobre práticas saudáveis realizadas pelo enfermeiro e por outros profissionais de saúde da Atenção Primária são, muitas vezes, desprezadas ou ignoradas pelos próprios familiares da criança, e os pais são os principais contribuintes para os filhos adquirirem hábitos e práticas saudáveis ${ }^{15-}$ 16,27. Na pesquisa realizada por Penn et al. (2014) foram convocados no total de 300 pais para participarem de um programa de intervenção na educação alimentar dos filhos. Deste total, somente 19 pais aceitaram comparecer. A negação dos pais em comparecem nas ações educativas representam um atraso nas intervenções preventivas.

Também foi citado nos estudos que a responsabilidade dos pais cuidarem dos filhos, é muitas vezes, repassada para outros, como babá, avós, tias e vizinho, devido a vida corriqueira no vínculo empregatício dentre outras ocupações no dia-a-dia17. A ausência dos pais nas ações educativas representa a segunda maior dificuldade na realização do aconselhamento nutricional.

Outra condição que dificulta a conduta do enfermeiro perante a obesidade infantil, é o elevado consumo de alimentos industrializados e estilo de vida sedentário da família. Segundo os relatos dos enfermeiros apontados no estudo realizado pelo autor Moura et al., (2015), os alimentos industrializados como: hambúrgueres, refrigerantes, biscoito e salgados empacotados, doces, macarrão instantâneo, dentre outros não nutritivos, são alimentos práticos de baixo custo e que possuem grande adesão pelas crianças e são muitas vezes oferecidos pelos pais como forma de recompensa.
Logo, a má alimentação em conjunto com a baixa adesão para práticas de atividades físicas, crescentemente substituída pela "tecnologia de conforto", como televisão, celulares, games, tablets, notebooks, entre outros aparelhos eletrônicos, são fortes influenciadores para a criança. $\bigcirc$ uso inadequado destes aparelhos traz prejuízos no desenvolvimento infantil, estimulando cada vez mais a vida sedentária 11,22.

Fato que faz o enfermeiro e outros profissionais da saúde enfrentarem várias crenças dos familiares para realizar a abordagem nutricional na infância. Tornando um conflito entre a experiência de vida dos pais com o conhecimento científico prestado. Essas crenças trazidas de uma pessoa a outra, tradições e valores ancorados em experiências de outros indivíduos, acarretam negativamente para a prestação e promoção da saúde, causando forte impacto nas orientações prestadas pela equipe de saúde trazendo receio e insegurança dos pais em seguilas ${ }^{11,15}$.

Outras dificuldades encontradas nos estudos estão relacionadas à inserção da mãe no mercado de trabalho, baixa escolaridade e mãe menor de idade. Pois essas mães, possuem maior incidência de não reconhecerem as necessidades da criança, o que leva a outra dificuldade encontrada nos estudos, o desmame precoce ${ }^{17}$.

- leite materno oferece inúmeros benefícios e tem a capacidade suficiente de suprir todas as necessidades nutricionais até o sexto mês de idade da criança ${ }^{23}$. Mas, o leite oferecido através de fórmulas lácteas e demais suplementos infantis são de fácil acesso, preparo e semelhante ao leite materno. Ambos fornecem energia, hidratação e nutrientes, mas, pela praticidade e custo acessivel para as mães, as fórmulas 
lácteas são comumente oferecidas para a criança antes de complementar os seis meses de vida ${ }^{17}$.

Outro ponto abordado no estudo de Moura et al. (2015), é o apoio financeiro oferecido pelo governo por meio do Bolsa Família, com a finalidade de não prejudicar o estado nutricional das crianças de famílias carentes de baixa renda financeira. Mas, por outras razões, grande parte dos pais focam em outras prioridades que impõem as necessidades nutricionais da criança, as oferecendo alimentos não nutritivos de baixo custo ${ }^{17}$.

Em relação à categoria, "Dificuldades para abordagem nutricional da criança", nota-se que é fundamental, o enfermeiro em conjunto com a equipe multidisciplinar em saúde da Atenção Primária, promova atividades de educação em saúde com a finalidade de estimular a participação dos pais e familiares nas atividades educativas. E respeitar as crenças, culturas, representações, ambientes nos quais estão inseridos, para que os pais ou responsáveis pela criança se tornem participantes ativos deste processo educativo e estimular seus sensos críticos quanto à mudança de suas práticas alimentares e comportamentais ${ }^{15,18}$.

$\mathrm{Na}$ segunda categoria, as dificuldades enfrentadas pelo enfermeiro para acompanhamento do crescimento e desenvolvimento infantil, os dois obstáculos mais citados nos estudos, estão relacionados a problemas de infraestrutura para prestação de serviços de saúde e falta de equipamentos e impressos para prestação dos cuidados e tratamento. A Unidade Básica de Saúde (UBS), segundo o Ministério da Saúde ${ }^{24}$, deve ser um ambiente tranquilo com uma estrutura ampla que proporcione aos profissionais de saúde e aos usuários segurança e praticidade no atendimento.
A falta de recursos e um espaço físico inadequado na UBS faz com que a assistência prestada do enfermeiro e demais profissionais de saúde ao usuário fique comprometida. E avaliar os dados antropométricos infantis é um dos indicadores em saúde mais importantes nas consultas de puericultura. Pois, a partir destes dados obtidos o enfermeiro consegue avaliar o estado nutricional, o crescimento e desenvolvimento da criança ${ }^{18,20,25}$.

Nas consultas de puericultura, o enfermeiro deve ir além de uma abordagem técnica simples, como, pesar, medir e prestar orientações básicas. Deve-se realizar uma consulta de enfermagem completa, composta por uma anamnese, exame físico, interpretação de dados, diagnósticos de enfermagem, prescrições com orientações e intervenções pertinentes. Orientar e transferir conhecimento aos familiares, tornando muitas vezes uma prática mais educativa que assistencial ${ }^{10 .}$

A escassez de informações nos registros nas evoluções de enfermagem representa o terceiro maior resultado para acompanhamento do crescimento e desenvolvimento. Segundo o autor Corgozinho et al., (2013), há muitas falhas nos registros de enfermagem, informações inespecíficas, e muitas dessas evoluções não seguem a padronização do Ministério da Saúde referentes a saúde da criança. Nas condutas e orientações da enfermagem, é comumente utilizado o termo "Orientações gerais", sem nenhuma especificação que contribua para melhor análise dos cuidados de enfermagem.

Os registros de enfermagem e de outros profissionais de saúde na Caderneta de Saúde da Criança são essenciais para o processo do cuidar, possibilita uma comunicação segura entre os 
profissionais, avaliação da qualidade da assistência prestada, processos éticos e judiciais e colaboração para o ensino e pesquisa. As informações sobre os diversos aspectos da saúde da criança são fundamentais para tornar a Caderneta da Criança um importante instrumento de vigilância, de promoção da saúde e de articulação entre a Unidade Básica de Saúde e outros serviços de saúde 26-27.

A falta de capacitações técnicas e científicas é outro obstáculo encontrado nos estudos para abordar com eficiência e qualidade o assunto obesidade infantil. Segundo o autor Penn et al (2014) é preciso que o enfermeiro seja simples e objetivo em suas orientações em saúde, ser cauteloso no momento da comunicação com os pais, usar termos como "excesso de peso" ao invés de "obeso".

Neste momento é essencial a participação da gestão em saúde juntamente com o enfermeiro para promoção das diretrizes educativas para incentivar e apoiar a mudança comportamental entre pais e filhos para práticas saudáveis e irem além da Unidade Básica de Saúde, como por exemplo, realizar ações educativas em escolas.

- Programa Saúde na Escola é responsável por ações de promoção e prevenção de saúde de forma integrada a Atenção Básica com professores, funcionários da escola e família dos alunos. As ações desenvolvem avaliação clínica, nutricional, vacinal e promoção de hábitos saudáveis. Neste momento, é necessário que o enfermeiro e gestores garantam espaço, instrumentos de trabalho, tempo para as atividades planejadas de orientação, educação e promoção de saúde. Dentro das disponibilidades e atividades programadas, o enfermeiro pode atuar nas ações educativas de forma isolada ou com uma ação interdisciplinar envolvendo outros profissionais da saúde 28-29.

Ensinar e orientar pais e crianças é um processo contínuo que exige do enfermeiro conhecimento, técnica, talento, dedicação, criatividade, sabedoria e perseverança. Apesar da importância das mudanças e adoção de hábitos saudáveis, o enfermeiro deve entender que cada família tem seu tempo, e somente eles podem decidir mudar. $O$ enfermeiro deve ter uma postura empática, tolerante e compreensiva, entendendo que hábitos de vida são arraigados ao longo de uma vida e que alterar hábitos pode ser um processo difícil e demorado, levando tempo para entender e adaptar com as mudanças.

\section{Conclusões}

Nota-se que as principais dificuldades enfrentadas pelo enfermeiro na prevenção da obesidade infantil estão relacionadas aos pais e na infraestrutura dos serviços de saúde e na falta de equipamentos para prestação dos cuidados de enfermagem.

O desconhecimento e desinteresse dos pais em relação à obesidade infantil é um grande desafio para o enfermeiro e demais profissionais de saúde. Neste contexto, é necessário criar estratégias e ações de saúde que envolvam os pais, enfermeiro e a equipe de saúde de forma dinâmica e educativa para um melhor entendimento dos fatores de riscos e um comprometimento maior nas medidas preventivas. 
No entanto, é indispensável a realização de capacitações e qualificações nos serviços de saúde relacionadas a obesidade infantil para todos os profissionais. Visto que, causam maior importância e impacto na família quando orientadas por um profissional de saúde qualificado e motivado para essas ações.

Para que a assistência do enfermeiro e equipe multidisciplinar seja integral à criança, é preciso que a gestão em saúde avalie as condições da UBS e colaborem com seus funcionários, os oferecendo recursos suficientes para exercerem suas funções de forma segura, qualificativa e eficiente. É preciso também, buscar prestar atividades além da Unidade de Saúde, acompanhando continuamente o desenvolvimento e crescimento da criança em sua infância através dos dados registrados na Caderneta em Saúde da Criança.

A realização deste estudo, possibilitou compreender melhor as principais dificuldades enfrentadas pelo enfermeiro diante a obesidade infantil. Perante a importância e relevância deste tema, é necessário a realização de outros estudos que investiguem a efetividade das intervenções e estratégias do enfermeiro para promoção da saúde da família e da criança correlacionados ao tema.

\section{Declaração de conflito de interesses}

O autor do artigo afirma que não se encontram em situações de conflito de interesse que possam influenciar o desenvolvimento do trabalho, tais como emissão de pareceres, propostas de financiamento, promoções ou participação em comitês consultivos ou diretivos, participação em estudos clínicos experimentais subvencionados; atuação como palestrante em eventos patrocinados; participação em conselho consultivo ou diretivo; comitês normativos de estudos científicos; recebimento de apoio institucional; propriedade de ações; participação em periódicos patrocinados, assim como qualquer relação financeira ou de outra natureza com pessoas ou organizações que possam influenciar o trabalho de forma inapropriada.

\section{Referências}

1- BRASIL. Ministério da Saúde. Secretaria de Atenção à Saúde. Departamento de Atenção Básica. Estratégias para o cuidado da pessoa com doença crônica: obesidade / Ministério da Saúde, Secretaria de Atenção à Saúde, Departamento de Atenção Básica. - Brasília : Ministério da Saúde, 2014. 212 p.: il. - (Cadernos de Atenção Básica, n. 38). Disponivel em:<http://bvsms.saude.gov.br/bvs/pu blicacoes/estrategias_cuidado_doenc a_cronica_obesidade_cab38.pdf>

2- LEAL, Vanessa Sá; LIRA, Pedro Israel Cabra de; OLIVEIRA, Risia C. E. de Menezes; SEQUEIRA, Leopoldina Augusta de Souza; NETO, Manoel Alexandre de Arruda; ANDRADE, Sonia Lúcia Lucena Sousa de; et al. Excesso de peso em crianças e adolescentes no Estado de Pernambuco, Brasil: prevalência e determinantes. Caderno Saúde Pública, Rio de Janeiro. Vol. 28, n. 6, p. 1175-1182, 2012.

3- FARIA, Ezequiel Patrício de; KANDLER, Ingrid; COUTINHO, Fábio 
Gonçalves. Obesidade Infantil no

Âmbito da Atenção Primária. Revista Inova Saúde, Criciúma, vol. 10, n. 2, jul. 2020.

4- OMS. Organização Mundial da Saúde. Número de crianças com excesso de peso pode chegar a 70 milhões até 2025, alerta OMS. 2015. Disponível em:

https://nacoesunidas.org/numero-decriancas-com-excesso-de-peso-podechegar-a-70-milhoes-ate-2025-alertaoms/.

5- ALMEIDA, Loureana de Melo; FORMIGA, Walnara Arnaud Moura; LIMA, Rafael Ferreira; SILVA, Waleska Gualberto da; SILVA, Isabela Letícia de Andrade e; SILVA, Sabrina Bezerra da; et al. (2020). Fatores associados ao sobrepeso e obesidade infantil. Revista Eletrônica Acervo Saúde, (58), e4406. https://doi.org/10.25248/reas.e4406.20 20.

6- SOUSA, Edmilson; GUERREIRO, Michelle. O papel do enfermeiro na obesidade infantil. Revista de Enfermagem Faciplac. Vol. 1. N. 1. 2016.

7- BOMFIM, Eliane dos Santos; ARAÚJO, Igor Brasil de; SANTOS, Adriana Glay Barbosa; SILVA, Adson Pereira; VILELAS, Alba Benemérita Alves; YARID, Ségio Donha. Atuação do enfermeiro acerca das práticas educativas na Estratégia Saúde e Família. Rev enferm UFPE on line., Recife, 11(Supl. 3):1398-402, mar., 2017.
DOI: 10.5205/reuol.10263-91568-1-

RV.1103sup201711.

8- ARAÚJO, Sarah Nilkece Mesquita; LUZ, Maria Helena Barros Araújo; ROCHA, Silvana Santiago da; SILVA, Grazielle Roberta Freitas da; DUARTE, Marianne Rocha; SANDES, Naiana Martins de. Obesidade infantil: conhecimentos e práticas de enfermeiros da Atenção Básica. Enfermagem em foco. Vol. 3, n. 03, p. 139-142, 2012.

9- OLIVEIRA, Genykléa Silva; BRAZ, Josineide do Nascimento; NASCIMENTO, Luzia Kelly Alves da Silva; MELO, Marina Clarissa Barros de. Obesidade Infantil: Contribuição da Enfermagem na Prevenção. Revista Humano Ser - UNIFACEX, NatalRN.Vol. 01, n.1, p 1-12, 2016.

10--ZANARDO, Graziani Maidana; ANDRADE, Ursulla; ZANARDO, Guilherme Maidana; MENEZES, Luana Possomai. Atuação do Enfermeiro na Consulta de Puericultura: Uma revisão Narrativa da Literatura. Revista de Enfermagem | FW | v. 13 | n. 13 | p. 55-69| 2017.

11- PALOMBO, Claudia Nery Teixeira; FUJIMORI, Elizabeth; TORIYAMA, Áurea Tamami Minagawa; DUARTE, Luciane Simões; BORGES, Ana Luiza Vilela. Difficulties in nutritional counseling and child growth followup: from a professional perspective. Revista Brasileira de Enfermagem [Internet]. 2017. [Thematic Edition "Good practices and fundamentals of Nursing work in the construction of a 
democratic society"]. Vol. 70. n. 5. p. 249-257.

12- SOUZA, Marcela Tavares de; SILVA, Michelly Dias da; CARVALHO, Rachel de. Revisão integrativa: o que é e como fazer. Einstein (São Paulo) [online]. 2010, vol.8, n.1, pp.102-106.

13- ECA. Estatuto da Criança e do Adolescente. Lei n 8.069, de 13 de julho de 1990.

Disponível:<https://presrepublica.jusb rasil.com.br/legislacao/91764/estatutoda-crianca-e-do-adolescente-lei8069-90>.

14- BRASIL. Ministério da Saúde. Conselho Nacional de Saúde. Resolução 466, de 12 de dezembro de 2012. Aprovar as seguintes diretrizes e normas regulamentadoras de pesquisas envolvendo seres humanos. Disponível em: $<$ https://bvsms.saude.gov.br/bvs/saud elegis/cns/2013/res0466_12_12_2012.ht $\mathrm{ml}>$.

15- WARREN, N.; HUNT, J. (2017). Barriers to addressing childhood weight issues in acute care settings and how to overcome them. Nursing Children and Young People. Vol: 29. n. 05. p.1823.

16- ANTI, Eliza; LAURENT, Jennifer S; TOMPKINS, Connie. The Health Care Provider's Experience With Fathers of Overweight and Obese Children: A Qualitative Analysis. Journal of Pediatric Health Care. Vol. 30, n. 02, 2016 p. 99-107.
17- MOURA, Mayara Águida Porfírio; ROCHA, Silvana Santiago da; $\mathrm{PINHO}$, Diana Lúcia Moura; GUILHEM, Dirce. Facilidades e dificuldades dos enfermeiros no cuidar da alimentação infantil na atenção básica. Artigo Original. Original Paper. O Mundo da Saúde, São Paulo -2015.Vol. 39, n. O2. p. 231-238.

18- PENN, Sarah; KERR, Joanne. Childhood obesity: the challenges for nurses. Nursing children and young people. 2014. Vol. 26, n. 02. p. 16-21.

19- CORGOZINHO, Juliana Nunes Costa; RIBEIRO, Gabriela de Cássia. Registros de Enfermagem e o enfoque na prevenção da obesidade infantil. Revista de Enfermagem do CentroOeste Mineiro. 2013 set/dez. Vol. 03, n. 03. p. 863-872.

20- ISMA, Gabriella E.; BRAMHAGEN, Ann-Cathrine; AHLSTROM, Gerd; OSTMAN, Margareta; DYKES, AnnaKarin. Obstacles to the prevention of overweight and obesity in the context of child health care in Sweden. BMC Family Practice 2013. Vol. 14, n.01. p. 143.

21- QUELLY, S. B. (2013). Influence of Perceptions on School Nurse Practices to Prevent Childhood Obesity. The Journal of School Nursing, Vol. 30, n. 04, p. 292-302.

22- PAIVA, Natália Moraes Nolêto; COSTA, Johnatan da Silva. A influência da tecnologia na infância: desenvolvimento ou ameaça?. Psicologia. pt, Vol. 01, p. 1-13, 2015. 
23- BRASIL. Ministério da Saúde. Secretaria de Atenção à Saúde.

Departamento de Atenção Básica.

Saúde da criança : aleitamento

materno e alimentação

complementar / Ministério da Saúde,

Secretaria de Atenção à Saúde,

Departamento de Atenção Básica. - 2 . ed. - Brasília : Ministério da Saúde,

2015. 184 p. : il. - (Cadernos de Atenção

Básica; n. 23). Disponível em: < https://bvsms.saude.gov.br/bvs/public acoes/saude_crianca_aleitamento_m aterno_cab23.pdfs.

24- BRASIL. Ministério da Saúde. Secretaria de Atenção à Saúde. Departamento de Atenção Básica. Manual de estrutura física das unidades básicas de saúde: saúde da família / Ministério da Saúde, Secretaria de Atenção à Saúde, Departamento de Atenção Básica - 2 . ed. - Brasília : Ministério da Saúde, 2008.52 p. : il. color - (Série A. Normas e Manuais Técnicos). Disponivel em: < http://bvsms.saude.gov.br/bvs/publica coes/manual_estrutura_fisica_ubs.pdf $>$.

\section{5- ALMEIDA, Luana Mirelle de;} CAMPOS, Kátia Ferreira Costa; RANDOW, Raquel; GUERRA, Vanessa de Almeida. Estratégias e desafios da gestão da atenção primária à saúde no controle e prevenção da obesidade. Revista Gestão \& Saúde (Brasília). Vol. 08, n. 01, Jan. 2017. p. 114139.
26- SILVA, Fabiane Blanco e; GAÍVA, Maria Aparecida Munhoz; MELLO, Débora Falleiros de. Utilização da Caderneta de Saúde da Criança pela Família: percepção dos profissionais. Texto Contexto Enfermagem, Florianópolis, 2015 Abr-Jun; 24(2): 407 14.

27- COFEN. Conselho Federal de Enfermagem. Guia de Recomendações para Registro de Enfermagem no prontuário do paciente e outros Documentos de Enfermagem. 2016. Disponível em: < http://www.cofen.gov.br/wpcontent/uploads/2016/08/Guia-deRecomenda\%C3\%A7\%C3\%B5esCTLN-Vers\%C3\%A3o-Web.pdf.>.

28- SANTOS, Fabiane Dias da Rocha dos; VITOLA, Camila Bueno; ARRIEIRA, Isabel Cristina de Oliveira; CHAGAS, Maria Cristina da Silveira; GOMES, Giovana Calcagno; PEREIRA, Fabiani Weiss. Ações de enfermeiros e professores na prevenção e no combate à obesidade infantil. Revista Rene. 15(3): 463-470, maio-jun. 2014.

29- OLIVEIRA, Ernani Coimbra de; ADÃO, Isabel Cristina; ROSA, Élida Ferreira Torga. Considerações sobre a enfermagem na escola e suas práticas educativas. Saberes Interdisciplinares, [S.I.], v. 10, n. 19, p. 29-40, nov. 2018. ISSN 2675-2255. 